Check for updates

The BMJ

Cite this as: BMJ 2021;375:n2422 http://dx.doi.org/10.1136/bmj.n2422 Published: 04 October 2021

\title{
Covid-19: Molnupiravir reduces risk of hospital admission or death by $50 \%$ in patients at risk, MSD reports
}

\section{Elisabeth Mahase}

The antiviral drug molnupiravir reduced the risk of admission to hospital or death by around 50\% in non-hospitalised adults who had mild to moderate covid-19 and were at risk of poor outcomes, Merck Sharp and Dohme (MSD) has reported. ${ }^{1}$

The company said in a press release that $7.3 \%$ of patients (28 of 385) who received molnupiravir as part of the phase III trial and $14.1 \%$ of patients taking placebo (53 of 377) either were admitted to hospital or had died by day 29 after randomisation.

At day 29 no deaths were reported in the molnupiravir group, while eight were reported in the placebo group. Recruitment to the trial has now been stopped on the advice of its independent data monitoring committee because of the positive results.

MSD and its partner Ridgeback Biotherapeutics intend to produce 10 million courses of treatment by the end of 2021, with more doses expected in 2022. The US has already agreed a deal for 1.7 million courses.

The interim analysis included 775 patients who were initially enrolled in the trial on or before 5 August 2021. To be eligible, all adults had to have confirmed mild or moderate covid-19, symptom onset within five days of study randomisation, and at least one risk factor associated with poor disease outcome.

Molnupiravir was found to reduce the risk of hospital admission or death across all key subgroups, with efficacy unaffected by the timing of symptom onset, underlying risk factors, or variant type (gamma, delta, and $\mathrm{mu}$ ), the press release said.

The company said the incidence of adverse events was similar in the groups (35\% in the molnupiravir group and $40 \%$ in the placebo group). Drug related adverse events were also similar (12\% molnupiravir, $11 \%$ placebo), although fewer people in the molnupiravir group discontinued treatment because of an adverse event $(1.3 \%)$ than in the placebo group (3.4\%).

MSD has said it planned to implement a "tiered pricing approach" based on World Bank country income criteria, and it has also entered into non-exclusive voluntary licensing agreements for the drug with established manufacturers of generics to accelerate availability in more than 100 low and middle income countries.

Peter Horby, professor of emerging infectious diseases at the University of Oxford, said, "The proportional reduction in the risk of hospitalisation or death is impressive, but it is important to remember that the absolute risks were $14 \%$ reduced to $7 \%$, so quite a lot of people need to be treated to prevent one hospitalisation or death. This means the drug needs to be very safe and affordable. The safety data also look good, so again this is encouraging."

However, he warned, "We need to watch for resistance and think from the outset about the potential role of combination antiviral therapies."

1 Merck and Ridgeback's investigational oral antiviral molnupiravir reduced the risk of hospitalization or death by approximately 50 percent compared to placebo for patients with mild or moderate covid-19 in positive interim analysis of phase 3 study. Oct 2021. https://www.merck.com/news/merckand-ridgebacks-investigational-oral-antiviral-molnupiravir-reduced-the-riskof-hospitalization-or-death-by-approximately-50-percent-compared-toplacebo-for-patients-with-mild-or-moderat.

This article is made freely available for use in accordance with BMJ's website terms and conditions for the duration of the covid-19 pandemic or until otherwise determined by BMJ. You may use, download and print the article for any lawful, non-commercial purpose (including text and data mining) provided that all copyright notices and trade marks are retained. 